\title{
Spike protein amino acid variants increased miRNA binding and hence reduced virulence of SARS-CoV-2 in Jordan, Middle East
}

\author{
Hazem Haddad ( $\square$ hazem_haddad1981@just.edu.jo ) \\ Princess Haya Biotechnology Centre, Jordan University of Science and Technology, Irbid, Jordan \\ Ramzi Foudeh \\ Jordanian Society of Genetic Engineers (JSGE), Amman, Jordan \\ Walid Al-Zyoud \\ Department of Biomedical Engineering, School of Applied Medical Sciences, German Jordanian University, Amman, Jordan
}

\section{Research Article}

Keywords: Jordan, miRNA, SARS-CoV-2 transmission

Posted Date: May 17th, 2020

DOI: https://doi.org/10.21203/rs.3.rs-28866/v1

License: @ (i) This work is licensed under a Creative Commons Attribution 4.0 International License. Read Full License

Version of Record: A version of this preprint was published on August 20th, 2020. See the published version at https://doi.org/10.1016/j.ncrna.2020.08.002. 


\section{Abstract}

Initial epidemiological studies inform the central COVID-19 disease prophet. Some papers have been studying miRNA and viral RNA interaction target predictions over the past few years. In this work, via the miRDB database, we determined the target scores of predicted miRNA to bind with the ss-RNA of SARS-CoV-2 in general and spike gene in specific. Our predicted miRNA targets of the ss-RNA of SARS-CoV-2 might destabilize and hence inhibit the ss-RNA translation of SARS-CoV-2 and prevent viral replication that has been established by more than $80 \%$ of asymptomatic infected cases in Jordan due to host miRNA interactions. In respiratory epithelial cells, the high scoring miRNAs prediction covers the RNA from 5 ' to $3^{\prime}$ that explain successful antiviral defenses against ss-RNA of SARS-CoV-2 and lead to new nucleotide deletion mechanisms. The exciting finding here that the substitution $1841 \mathrm{~A}>\mathrm{G}$ at the viral genomic RNA level or the D614G at spike protein level showed a change in the predicted miRNA sequence and an increase in the target score (from 91 to 92 ) (hsa-miR4793-5p to hsa-miR-3620-3p).

\section{Introduction}

Severe acute respiratory syndrome coronavirus 2 (SARS-CoV-2) caused an outbreak in Wuhan city and characterized as a pandemic by the World Health Organization WHO [1]. The first case of SARS-CoV-2 was reported to the Jordanian Ministry of Health on March 2, 2020, for a Jordanian citizen who was in Italy. To the date of this report, there are 522 confirmed cases, 387 recovered and nine deaths of COVID-19 in Jordan, according to the Jordanian Ministry of Health official via its web site launched as a unified source of information about the preventive measurements and symptoms of corona virus-2019 (https://corona.moh.gov.jo/en).

In addition, 18 years ago, severe coronavirus acute breathing syndrome (SARS-CoV) appeared in China. It has spread to over 30 countries, infecting around 8,000 people, killing young people $10 \%$, and aging $50 \%$. No SARS CoV or other up-and-coming CoV vaccines or antivirals such as Middle East respiratory CoV (MERS-CoV) are approved to date [2]. The molecular mechanisms of viral pathogenesis will provide thoughtful help in the search for effective and secure therapeutic strategies against new and well-known human CoVs.

MicroRNAs (miRNAs) are non-coding RNAs that control many function targets within a cell by controlling protein levels through binding to mRNA translation process or mRNA abundance. Many evidence shows that miRNAs container the RNA virus replication and pathogenesis through direct binding to the RNA virus-mediated and changes in the host transcriptome. Proof of host miRNAs can bind to a wide-ranging of RNA viruses, straight adaptable their pathogenesis through mimics' cellular mRNAs tolerating direct binding of the miRNA to the viral RNA. Theoretically, the regulation is analogous to that of host mRNAs [3,4].

Many miRNA targeted in Influenza viral RNA segments were linked with the activity of host miRNA-induced antiviral defense. This represents potential Treatment with a combination of the five miRNAs through agomir delivery to suppressed viral replication and effectively improved protection against lethal challenge with PR8 in mice [5].

In previous study, severe acute coronavirus syndrome (SARS-CoV) causes human fatal disease and reaction and extensive pulmonary disease. The significance of small non-coding RNAs for SARS-CoV pathologies, sequenced lung RNAs of infected mouse, and three 18 to 22 t small viral RNAs (SvRNAs), were discovered. The three svRNAs originated from the SARS-CoV genomic regions nsp3 (svRNA-nsp3.1 and -nsp3.2), and N (svRNA-N). CoV svRNAs were autonomous, cell type and host species from RNase III; but the extent of viral replication was dependent. In vivo lung pathology and pro-inflammatory cytokine release, antagomir-mediated inhibition of svRNA-N significantly decreased. This indicates that SvRNAs contribute to the pathogenesis of SARS-CoV and demonstrates the potential for antagomers of svRNA-N as antivirals [6].

To understand the early steps of COVID-19 infection, we predicted miRNAs targeting the submitted 29903 bp ss-RNA of the severe acute respiratory syndrome coronavirus 2 (SARS-CoV-2 sequence), the isolate of Wuhan-Hu-1, complete genome. A predicted miRNAs targeting region at 3822 bp ss-RNA of the spike glycoprotein of SARS-CoV-2 was revealed. Also, we predicted miRNAs targeting a variable region of the ss-RNA spike glycoprotein of SARS-CoV-2 sequences from 20 positive nasopharyngeal specimens. These specimens were collected in Jordan and sequenced by Biolab Diagnostic Laboratories (Jordan) \& Andersen lab at Scripps Research (USA), who deposited the sequences in GISAID, a maintained global database based in Germany. The perception in this work might help scientists to understand the molecular mechanisms of viral pathogenesis. In addition, it might support the research for effective, safe therapeutic strategies against known human CoVs and new emergent strains with a focus on miRNA-induced antiviral human body defense, which could be a potential treatment development for SARS-CoV-2.

\section{Methods}

The miRDB is an online http:/mirdb.org/custom.html database for the target and working annotations of miRNA. All targets in miRDB were anticipated by MirTarget, a bioinformatics tool that was developed through the study of thousands of miRNA target interactions from high-performance research [7,8].Using http:/biomodel.uah.es/en/lab/cybertory/analysis/trans.htm to convert sequences from miRNA to DNA to demonstrate genome sequence alignment using ChromosPros Version 2.1.9. Wuhan-Hu-1, full NC 045512.2 accession genome sequence number and MIRNAs targeting area 3822 bp ss-RNA-spike SARs-CoV2 Spike. We predicted miRNA targets 29903 bp ss-RNA (SARS-CoV-2 sequence) isolate Wuhan-Hu-1. In addition, miRNAs are predicted to target region on the SARs-CoV-2 sequence Accession no YP 009724390 ss-RNA spike glycoprotein variability from Jordanian positive nasopharyngeal specimens sequenced by Biolab Diagnostic Laboratories (Jordan) \& Andersen lab at Scripps Research (USA) who published sequences retrieved from GISAID

(https://corona.moh.gov.jo/en) (https://gisaid.org).

\section{Results And Discussion}


We predicted top ten score miRNAs targeting between (98-99) the submitted 29903 bp ss-RNA SARS-CoV-2 genome, accession number NC_045512.2, correspondingly, hsa-miR-4288, hsa-miR-195-5p, hsa-miR-16-5p, hsa-miR-15b-5p, hsa-miR-15a-5p, hsa-miR-6838-5p, hsa-miR-497-5p, hsa-miR-424-5p, hsamiR-3133, hsa-miR-21-3p Table 1. These miRNAs are presented in miRDB, along with associated function annotations. As a recent update, miRDB displays expressions of hundreds of cell lines, and the user may limit their search for the cell line of interest miRNA targets. miRDB offers an integrative analysis of the target prediction and gene ontological data found in Tables 2,3,4,5,6,7,8,9,10 and 11, to promote the prediction of miRNA functions.In addition, we predicted only three miRNAs (hsa-miR-510-3p, hsa-miR-624-5p, hsa-miR-497-5p) with targeting scores of (92,90 and 84 ) respectively for the submitted 3822 bp of the ss-RNA spike glycoprotein of SARS-CoV-2 sequence of Wuhan-Hu-1 (from the complete genome NCBI reference sequence: NC_045512.2 Region: 21563-

25384) as shown in Table 13. These miRNAs, as well as associated functional annotations, presented in miRDB presents the expression profiles of hundreds of cell lines. To facilitate the prediction of miRNA functions, miRDB offers for integrative analysis of target prediction and Gene Ontology data found in Tables 14 and 15.

Moreover, in our study here we predicted the score of miRNAs targeting regions on many ss-RNA spike glycoprotein of SARS-CoV-2 sequence from Jordanian samples with amino acid substitutions (NCBI Reference Sequence: NC_045512.2 region: 21563-25384 and Nomenclature sequence, Amino Acid Variant (SAV) and annotation used the accession number YP_009724390.1).

The original sequence of the del 432TTA, and the del Y144 have the same miRNA with a target score of 91. The original 1841A target miRNA score (91, 64 and 56) for (hsa-miR-4793-5p, hsa-miR-143-5p and hsa-miR-3133) respectively. The interesting finding here that the $1841 \mathrm{~A}>\mathrm{G}$, and $\mathrm{D} 614 \mathrm{G}$ showed a change in the predicted miRNA and an increase in the target score (from 91 to 92) (hsa-miR-4793-5p to hsa-miR-3620-3p). However, the original and 3415G>T D1139Y showed the same sequence of the miRNA (hsa-miR-548g-3p) and an increase in the target score of 80 to 81 . The last substitution of $3499 \mathrm{G}>\mathrm{A} \mathrm{G} 1167 \mathrm{~S}$ showed the same the miRNA sequence of (hsa-miR-155-5p) and a decrease in the target score from 73 to 72 .One of the record genomic changes observed in the severe acute respiratory syndrome coronavirus (SARS-CoV-1) isolated from humans after transmission human to human was the acquisition of a specific 29-nucleotide deletion occurred in open reading frame 8 (ORF8). Three top target score of miRNAs prediction (hsa-miR-497-5p, hsa-miR-195-5p and hsa-miR21-3p) showed on Table 12, 16 and 24 have an expression in the respiratory epithelial cells and effective antiviral defenses against the ss-RNA of SARS-CoV-2 and lead to new mechanisms interaction binding miRNA and SARS-CoV-2 of nucleotide deletion [5-6]\& [9].

\section{Conclusion}

Over the past few years, some articles studied the target prediction of miRNA and viral RNA interaction. In our predictions, more than $80 \%$ asymptomatic of the disease raised due to the host miRNA interactions, which have identified the target where the genome replication of the ss-RNA of SARS-CoV-2 has changed to inhibit the translation of the ss-RNA and hence preventing viral replication and stabilization by subsequent generations. The top target score of miRNAs prediction cover from ' 5' to $3^{\prime}$ in the respiratory epithelial cells that might be the reason for an effective antiviral defense against the ss-RNA of SARS-CoV-2 and lead to new mechanisms of nucleotide deletion in the coding region of a protein. The miRNAs found in all tissues have target gene functions, which lead to an identification of novel cellular pathways to block RNA viral replication or even host cell-specific targeting the regulation for the ss-RNA of SARS-CoV-2.

\section{Tables}

Table 1: Predicted miRNAs targeting the submitted 29903 bp ss-RNA SARS-COV-2 genome, accession number NC_045512.2

Top of Form

Bottom of Form

\begin{tabular}{|c|c|c|}
\hline \multicolumn{2}{|c|}{ Target Rank Target Score } & \multirow{2}{*}{$\begin{array}{l}\text { miRNA Name } \\
\text { hsa-miR-4288 }\end{array}$} \\
\hline 1 & 99 & \\
\hline 2 & 99 & hsa-miR-195-5p \\
\hline 3 & 99 & hsa-miR-16-5p \\
\hline 4 & 99 & hsa-miR-15b-5p \\
\hline 5 & 99 & hsa-miR-15a-5p \\
\hline 6 & 99 & hsa-miR-6838-5p \\
\hline 7 & 98 & hsa-miR-497-5p \\
\hline 8 & 98 & hsa-miR-424-5p \\
\hline 9 & 98 & hsa-miR-3133 \\
\hline 10 & 98 & hsa-miR-21-3p \\
\hline
\end{tabular}

Table 2: Predicted targets function for hsa-miR-4288 on 29903 bp ss-RNA SARS-COV-2 genome 


\begin{tabular}{ccccc}
\hline \multicolumn{5}{c}{ Target Rank Target Score miRNA Name Gene Symbol Gene Description } \\
\hline 1 & 97 & hsa-miR-4288 & UIMC1 & ubiquitin interaction motif containing 1 \\
2 & 97 & hsa-miR-4288 & CC2D1A & coiled-coil and C2 domain containing 1A \\
3 & 96 & hsa-miR-4288 & ING3 & inhibitor of growth family member 3 \\
4 & 96 & hsa-miR-4288 & KCMF1 & potassium channel modulatory factor 1 \\
5 & 96 & hsa-miR-4288 & CAPZB & capping actin protein of muscle Z-line subunit beta \\
6 & 96 & hsa-miR-4288 & BCL11A & BCL11A, BAF complex component \\
7 & 96 & hsa-miR-4288 & AMELX & amelogenin X-linked \\
8 & 96 & hsa-miR-4288 & NTM & neurotrimin \\
9 & 95 & hsa-miR-4288 & LRRC4C & leucine rich repeat containing 4C \\
10 & 95 & hsa-miR-4288 & PSIP1 & PC4 and SFRS1 interacting protein 1 \\
\hline
\end{tabular}

Table 3: Predicted targets function for hsa-miR-195-5p on 29903 bp ss-RNA SARS-COV-2 genome

\begin{tabular}{ccccc}
\hline \multicolumn{1}{c}{ Target Rank Target Score miRNA Name } & Gene Symbol Gene Description \\
\hline 1 & 100 & hsa-miR-195-5p & PAPPA & pappalysin 1 \\
2 & 100 & hsa-miR-195-5p & FASN & fatty acid synthase \\
3 & 100 & hsa-miR-195-5p & UNC80 & unc-80 homolog, NALCN channel complex subunit \\
4 & 100 & hsa-miR-195-5p & FGF2 & fibroblast growth factor 2 \\
5 & 100 & hsa-miR-195-5p & TNRC6B & trinucleotide repeat containing 6B \\
6 & 100 & hsa-miR-195-5p & PTPN4 & protein tyrosine phosphatase, non-receptor type 4 \\
7 & 100 & hsa-miR-195-5p & PHF19 & PHD finger protein 19 \\
8 & 100 & hsa-miR-195-5p & DESI1 & desumoylating isopeptidase 1 \\
9 & 99 & hsa-miR-195-5p & UBE2Q1 & ubiquitin conjugating enzyme E2 Q1 \\
10 & 99 & hsa-miR-195-5p & LSM11 & LSM11, U7 small nuclear RNA associated \\
\hline
\end{tabular}

Table 4: Predicted targets function for hsa-miR-16-5p on 29903 bp ss-RNA SARS-COV-2 genome

\begin{tabular}{ccccl}
\hline \multicolumn{5}{c}{ Target Rank Target Score miRNA Name Gene Symbol Gene Description } \\
\hline 1 & 100 & hsa-miR-16-5p & PAPPA & pappalysin 1 \\
2 & 100 & hsa-miR-16-5p & FASN & fatty acid synthase \\
3 & 100 & hsa-miR-16-5p & UNC80 & unc-80 homolog, NALCN channel complex subunit \\
4 & 100 & hsa-miR-16-5p & FGF2 & fibroblast growth factor 2 \\
5 & 100 & hsa-miR-16-5p & TNRC6B & trinucleotide repeat containing 6B \\
6 & 100 & hsa-miR-16-5p & PTPN4 & protein tyrosine phosphatase, non-receptor type 4 \\
7 & 100 & hsa-miR-16-5p & PHF19 & PHD finger protein 19 \\
8 & 100 & hsa-miR-16-5p & DESI1 & desumoylating isopeptidase 1 \\
9 & 99 & hsa-miR-16-5p & UBE2Q1 & ubiquitin conjugating enzyme E2 Q1 \\
10 & 99 & hsa-miR-16-5p & LSM11 & LSM11, U7 small nuclear RNA associated \\
\hline & & & &
\end{tabular}

Table 5: Predicted targets function for hsa-miR-15b-5p on 29903 bp ss-RNA SARS-COV-2 genome 


\begin{tabular}{ccccc}
\hline Target Rank Target Score miRNA Name Gene Symbol Gene Description \\
\hline 1 & 100 & hsa-miR-15b-5p & PAPPA & pappalysin 1 \\
2 & 100 & hsa-miR-15b-5p & FASN & fatty acid synthase \\
3 & 100 & hsa-miR-15b-5p & UNC80 & unc-80 homolog, NALCN channel complex subunit \\
4 & 100 & hsa-miR-15b-5p & FGF2 & fibroblast growth factor 2 \\
5 & 100 & hsa-miR-15b-5p & TNRC6B & trinucleotide repeat containing 6B \\
6 & 100 & hsa-miR-15b-5p & PTPN4 & protein tyrosine phosphatase, non-receptor type 4 \\
7 & 100 & hsa-miR-15b-5p & PHF19 & PHD finger protein 19 \\
8 & 100 & hsa-miR-15b-5p & DESI1 & desumoylating isopeptidase 1 \\
9 & 99 & hsa-miR-15b-5p & UBE2Q1 & ubiquitin conjugating enzyme E2 Q1 \\
10 & 99 & hsa-miR-15b-5p & LSM11 & LSM11, U7 small nuclear RNA associated \\
\hline
\end{tabular}

Table 6: Predicted targets function for hsa-miR-15a-5p on 29903 bp ss-RNA SARS-COV-2 genome

\begin{tabular}{ccccl}
\hline \multicolumn{1}{l}{ Target Rank Target Score } & miRNA Name & Gene Symbol Gene Description \\
\hline 1 & 100 & hsa-miR-15a-5p & PAPPA & pappalysin 1 \\
2 & 100 & hsa-miR-15a-5p & FASN & fatty acid synthase \\
3 & 100 & hsa-miR-15a-5p & UNC80 & unc-80 homolog, NALCN channel complex subunit \\
4 & 100 & hsa-miR-15a-5p & FGF2 & fibroblast growth factor 2 \\
5 & 100 & hsa-miR-15a-5p & TNRC6B & trinucleotide repeat containing 6B \\
6 & 100 & hsa-miR-15a-5p & PTPN4 & protein tyrosine phosphatase, non-receptor type 4 \\
7 & 100 & hsa-miR-15a-5p & PHF19 & PHD finger protein 19 \\
8 & 100 & hsa-miR-15a-5p & DESI1 & desumoylating isopeptidase 1 \\
9 & 99 & hsa-miR-15a-5p & UBE2Q1 & ubiquitin conjugating enzyme E2 Q1 \\
10 & 99 & hsa-miR-15a-5p & LSM11 & LSM11, U7 small nuclear RNA associated \\
\hline
\end{tabular}

Table 7: Predicted targets function for hsa-miR-6838-5p on 29903 bp ss-RNA SARS-COV-2 genome

\begin{tabular}{ccccc}
\hline \multicolumn{1}{l}{ Target Rank Target Score } & miRNA Name & Gene Symbol Gene Description \\
\hline 1 & 100 & hsa-miR-6838-5p & PAPPA & pappalysin 1 \\
2 & 100 & hsa-miR-6838-5p & FASN & fatty acid synthase \\
3 & 100 & hsa-miR-6838-5p & UNC80 & unc-80 homolog, NALCN channel complex subunit \\
4 & 100 & hsa-miR-6838-5p & FGF2 & fibroblast growth factor 2 \\
5 & 100 & hsa-miR-6838-5p & TNRC6B & trinucleotide repeat containing 6B \\
6 & 100 & hsa-miR-6838-5p & PTPN4 & protein tyrosine phosphatase, non-receptor type 4 \\
7 & 100 & hsa-miR-6838-5p & PHF19 & PHD finger protein 19 \\
8 & 99 & hsa-miR-6838-5p & UBE2Q1 & ubiquitin-conjugating enzyme E2 Q1 \\
9 & 99 & hsa-miR-6838-5p & LSM11 & LSM11, U7 small nuclear RNA associated \\
10 & 99 & hsa-miR-6838-5p & ANKUB1 & ankyrin repeat and ubiquitin domain containing 1 \\
\hline
\end{tabular}




\begin{tabular}{ccccc}
\hline \multicolumn{1}{c}{ Target Rank Target Score miRNA Name } & Gene Symbol Gene Description \\
\hline 1 & 100 & hsa-miR-497-5p & PAPPA & pappalysin 1 \\
2 & 100 & hsa-miR-497-5p & FASN & fatty acid synthase \\
3 & 100 & hsa-miR-497-5p & UNC80 & unc-80 homolog, NALCN channel complex subunit \\
4 & 100 & hsa-miR-497-5p & FGF2 & fibroblast growth factor 2 \\
5 & 100 & hsa-miR-497-5p & TNRC6B & trinucleotide repeat containing 6B \\
6 & 100 & hsa-miR-497-5p & PTPN4 & protein tyrosine phosphatase, non-receptor type 4 \\
7 & 100 & hsa-miR-497-5p & PHF19 & PHD finger protein 19 \\
8 & 99 & hsa-miR-497-5p & UBE2Q1 & ubiquitin conjugating enzyme E2 Q1 \\
9 & 99 & hsa-miR-497-5p & LSM11 & LSM11, U7 small nuclear RNA associated \\
10 & 99 & hsa-miR-497-5p & ANKUB1 & ankyrin repeat and ubiquitin domain containing 1 \\
\hline
\end{tabular}

Table 9: Predicted targets function for hsa-miR-424-5p on 29903 bp ss-RNA SARS-COV-2 genome.

\begin{tabular}{ccccc}
\hline \multicolumn{1}{c}{ Target Rank Target Score miRNA Name } & Gene Symbol Gene Description \\
\hline 1 & 100 & hsa-miR-424-5p & PAPPA & pappalysin 1 \\
2 & 100 & hsa-miR-424-5p & FASN & fatty acid synthase \\
3 & 100 & hsa-miR-424-5p & UNC80 & unc-80 homolog, NALCN channel complex subunit \\
4 & 100 & hsa-miR-424-5p & FGF2 & fibroblast growth factor 2 \\
5 & 100 & hsa-miR-424-5p & TNRC6B & trinucleotide repeat containing 6B \\
6 & 100 & hsa-miR-424-5p & PTPN4 & protein tyrosine phosphatase, non-receptor type 4 \\
7 & 100 & hsa-miR-424-5p & PHF19 & PHD finger protein 19 \\
8 & 99 & hsa-miR-424-5p & UBE2Q1 & ubiquitin-conjugating enzyme E2 Q1 \\
9 & 99 & hsa-miR-424-5p & LSM11 & LSM11, U7 small nuclear RNA associated \\
10 & 99 & hsa-miR-424-5p & ANKUB1 & ankyrin repeat and ubiquitin domain containing 1 \\
\hline
\end{tabular}

Table 10: Predicted targets function for hsa-miR-3133 on 29903 bp ss-RNA SARS-COV-2 genome.

\begin{tabular}{ccccc}
\hline \multicolumn{5}{c}{ Target Rank Target Score miRNA Name Gene Symbol Gene Description } \\
\hline 1 & 100 & hsa-miR-3133 & HOOK3 & hook microtubule tethering protein 3 \\
2 & 100 & hsa-miR-3133 & RIMS2 & regulating synaptic membrane exocytosis 2 \\
3 & 100 & hsa-miR-3133 & TGFBRAP1 & transforming growth factor-beta receptor-associated protein 1 \\
4 & 99 & hsa-miR-3133 & RPRD1A & regulation of nuclear pre-mRNA domain-containing 1A \\
5 & 99 & hsa-miR-3133 & TFAP2B & transcription factor AP-2 beta \\
6 & 99 & hsa-miR-3133 & PTPRK & protein tyrosine phosphatase, receptor type K \\
7 & 99 & hsa-miR-3133 & MAP2 & microtubule-associated protein 2 \\
8 & 99 & hsa-miR-3133 & NRF1 & nuclear respiratory factor 1 \\
9 & 99 & hsa-miR-3133 & KAT6A & lysine acetyltransferase 6A \\
10 & 99 & hsa-miR-3133 & URI1 & URI1, prefoldin like chaperone \\
\hline
\end{tabular}

Table 11: Predicted targets function for hsa-miR-21-3p on 29903 bp ss-RNA SARS-COV-2 genome. 


\begin{tabular}{ccccc}
\hline \multicolumn{1}{c}{ Target Rank Target Score miRNA Name Gene Symbol Gene Description } \\
\hline 1 & 99 & hsa-miR-21-3p & STK38L & serine/threonine kinase 38 like \\
2 & 98 & hsa-miR-21-3p & PCDH19 & protocadherin 19 \\
3 & 96 & hsa-miR-21-3p & LAMP1 & lysosomal associated membrane protein 1 \\
4 & 96 & hsa-miR-21-3p & GRIA2 & glutamate ionotropic receptor AMPA type subunit 2 \\
5 & 96 & hsa-miR-21-3p & TOGARAM1 & TOG array regulator of axonemal microtubules 1 \\
6 & 96 & hsa-miR-21-3p & ATP1B1 & ATPase Na+/K+ transporting subunit beta 1 \\
7 & 96 & hsa-miR-21-3p & TSC22D2 & TSC22 domain family member 2 \\
8 & 96 & hsa-miR-21-3p & NAP1L5 & nucleosome assembly protein 1 like 5 \\
9 & 95 & hsa-miR-21-3p & UBE4B & ubiquitination factor E4B \\
10 & 95 & hsa-miR-21-3p & ZNF326 & zinc finger protein 326 \\
\hline
\end{tabular}

Table 12: Predicted miRNAs targeting region on $29903 \mathrm{nt}$ SARS-COV-2 complete genome 


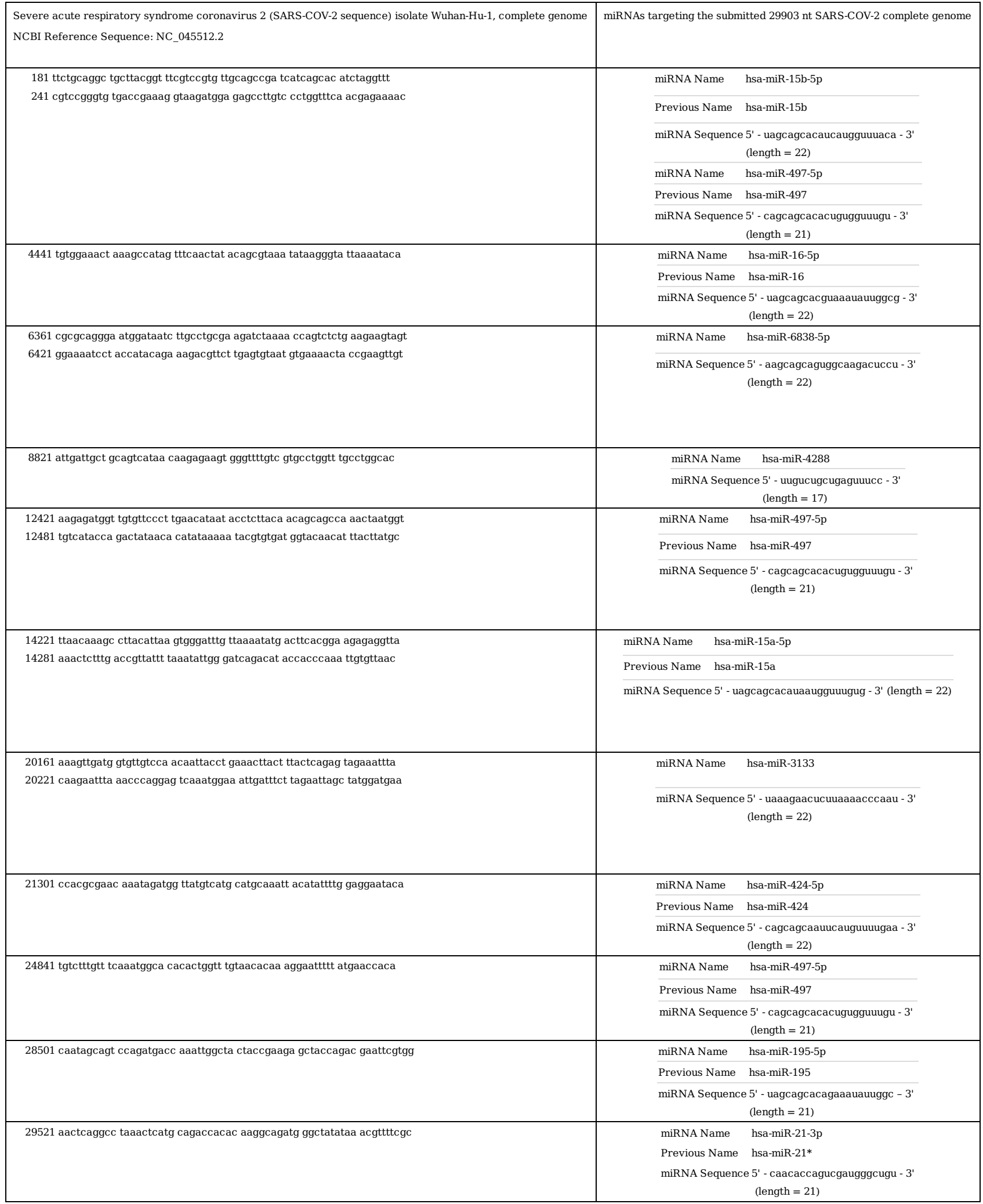




\begin{tabular}{ccc}
\hline \multicolumn{3}{c}{ Target Rank Target Score miRNA Name } \\
\hline 1 & 92 & hsa-miR-510-3p \\
2 & 90 & hsa-miR-624-5p \\
14 & 84 & hsa-miR-497-5p \\
\hline
\end{tabular}

Table 14: Predicted targets function for hsa-miR-510-3p on 3822 bp ss-RNA spike glycoprotein of SARS-COV-2 sequence, accession number NC_045512.2 REGION: $21563-25384$.

\begin{tabular}{ccccc}
\hline \multicolumn{1}{c}{ Target Rank Target Score miRNA Name } & Gene Symbol Gene Description \\
\hline 1 & 100 & hsa-miR-510-3p & CNOT6 & CCR4-NOT transcription complex subunit 6 \\
2 & 99 & hsa-miR-510-3p & NEXMIF & neurite extension and migration factor \\
3 & 99 & hsa-miR-510-3p & RBMS3 & RNA binding motif single stranded interacting protein 3 \\
4 & 99 & hsa-miR-510-3p & DENND6A & DENN domain containing 6A \\
5 & 99 & hsa-miR-510-3p & SNAP91 & synaptosome associated protein 91 \\
6 & 99 & hsa-miR-510-3p & BCLAF1 & BCL2 associated transcription factor 1 \\
7 & 99 & hsa-miR-510-3p & LATS2 & large tumor suppressor kinase 2 \\
8 & 99 & hsa-miR-510-3p & ELOVL7 & ELOVL fatty acid elongase 7 \\
9 & 98 & hsa-miR-510-3p & ZFHX3 & zinc finger homeobox 3 \\
10 & 98 & hsa-miR-510-3p & FMR1 & fragile X mental retardation 1 \\
\hline
\end{tabular}




\begin{tabular}{ccccc}
\hline \multicolumn{1}{c}{ Target Rank Target Score miRNA Name Gene Symbol Gene Description } \\
\hline 1 & 96 & hsa-miR-624-5p & ARL4A & ADP ribosylation factor like GTPase 4A \\
2 & 96 & hsa-miR-624-5p & SOWAHC & sosondowah ankyrin repeat domain family member C \\
\hline 3 & 95 & hsa-miR-624-5p & SYT1 & synaptotagmin 1 \\
\hline 4 & 95 & hsa-miR-624-5p & CLOCK & clock circadian regulator \\
\hline 5 & 95 & hsa-miR-624-5p & STK38 & serine/threonine kinase 38 \\
\hline 6 & 95 & hsa-miR-624-5p & LCP1 & lymphocyte cytosolic protein 1 \\
\hline 7 & 95 & hsa-miR-624-5p & ZNF800 & zinc finger protein 800 \\
\hline 8 & 95 & hsa-miR-624-5p & DOCK11 & dedicator of cytokinesis 11 \\
\hline 9 & 94 & hsa-miR-624-5p & UAP1 & UDP-N-acetylglucosamine pyrophosphorylase 1 \\
\hline 10 & 94 & hsa-miR-624-5p & NFKBIA & NFKB inhibitor alpha \\
\hline
\end{tabular}

Table 16: Predicted miRNAs targeting region on 3822 bp ss-RNA spike glycoprotein of SARS-COV-2 sequence

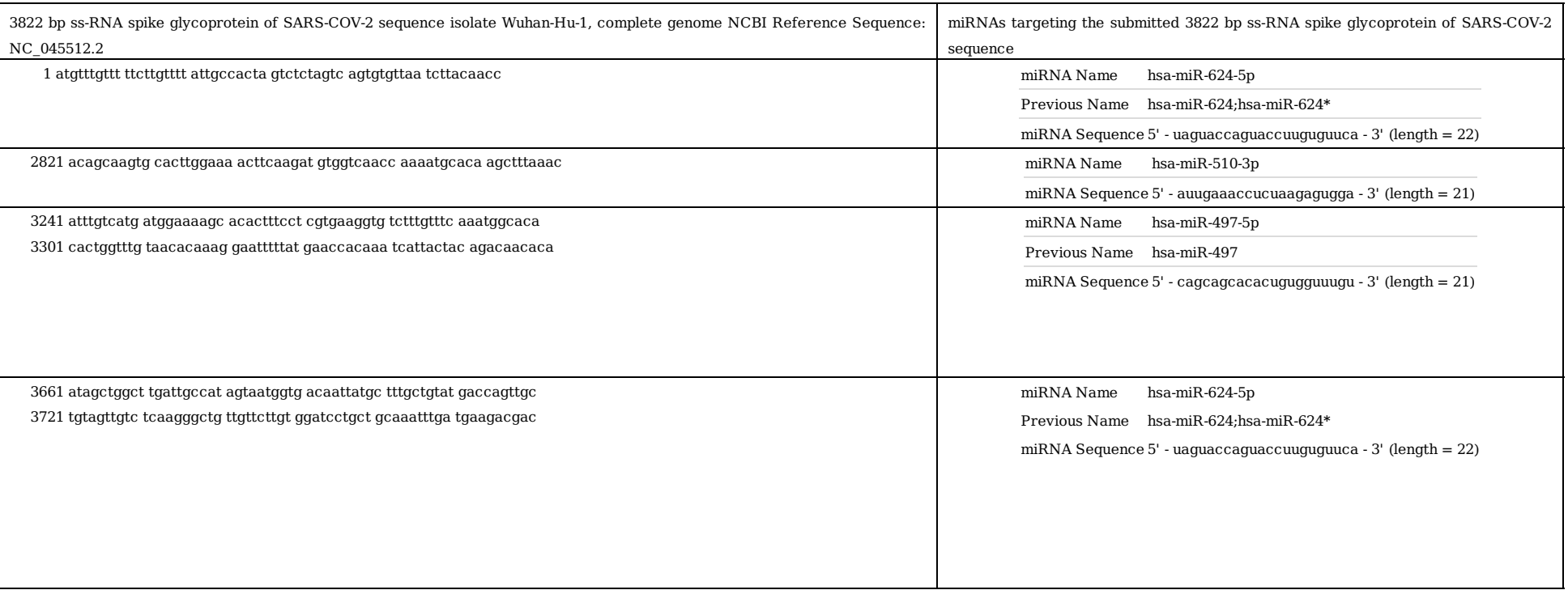

Table 17: Original and del 432 TTAdel. Y144 have the same miRNA and target score

\begin{tabular}{ccc}
\hline Target Rank Target Score & miRNA Name \\
\hline 1 & 91 & hsa-miR-196a-1-3p \\
\hline
\end{tabular}

Table 18: Original 1841 A target miRNA score

\begin{tabular}{ccc}
\hline Target Rank Target Score & miRNA Name \\
\hline 1 & 91 & hsa-miR-4793-5p \\
2 & 64 & hsa-miR-143-5p \\
3 & 56 & hsa-miR-3133 \\
\hline
\end{tabular}

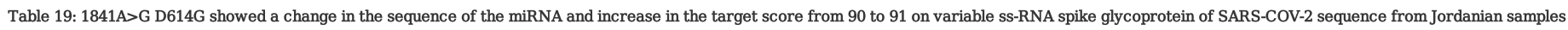

\begin{tabular}{|c|c|c|}
\hline \multicolumn{3}{|c|}{ Target Rank Target Score miRNA Name } \\
\hline 1 & 92 & hsa-miR-3620-3p \\
\hline 2 & 55 & hsa-miR-3133 \\
\hline 3 & 54 & hsa-miR-21-3p \\
\hline
\end{tabular}

Page $10 / 13$ 
Table 20: Original 3415G target miRNA score

\begin{tabular}{|c|c|c|}
\hline \multicolumn{3}{|c|}{ Target Rank Target Score miRNA Name } \\
\hline 1 & 80 & hsa-miR-548g-3p \\
\hline 2 & 72 & hsa-miR-627-5p \\
\hline 3 & 61 & hsa-miR-506-5p \\
\hline
\end{tabular}

Table 21: 3415G>T D1139Y show same the miRNA and increase the target score 80 to 81 on variation ss-RNA spike glycoprotein of SARS-COV-2 sequence from Jordanian samples

\begin{tabular}{|c|c|c|}
\hline \multicolumn{3}{|c|}{ Target Rank Target Score } \\
\hline 1 & 81 & hsa-miR-548g-3p \\
\hline 2 & 72 & hsa-miR-627-5p \\
\hline 3 & 61 & hsa-miR-506-5p \\
\hline
\end{tabular}

Table 22: Original $3499 \mathrm{G}$ target miRNA score.

\begin{tabular}{|c|c|c|}
\hline \multicolumn{2}{|c|}{ Target Rank Target Score } & \multirow{2}{*}{$\frac{\text { miRNA Name }}{\text { hsa-miR-155-5p }}$} \\
\hline 1 & 73 & \\
\hline 2 & 62 & hsa-miR-6765-5p \\
\hline 3 & 53 & hsa-miR-551b-5p \\
\hline
\end{tabular}

Table 23: 3499 G>A G1167S show same the miRNA and decrease the target score 73 to 72 on variation ss-RNA spike glycoprotein of SARS-COV-2 sequence from Jordanian samples

\begin{tabular}{ccc}
\hline Target Rank Target Score & miRNA Name \\
\hline 1 & 72 & hsa-miR-155-5p \\
2 & 62 & hsa-miR-6765-5p \\
3 & 51 & hsa-miR-668-3p
\end{tabular}

Table 24: Predicted miRNAs targeting region on variation ss-RNA spike glycoprotein of SARS-COV-2 sequence from Jordanian samples 


\begin{tabular}{|c|c|c|c|}
\hline $\begin{array}{l}\text { Sample number with accision } \\
\text { on GISAID }\end{array}$ & Sequence variation ss-RNA spike glycoprotein of SARS-COV-2 sequence & $\begin{array}{l}\text { Single } \\
\text { Amino } \\
\text { Acid } \\
\text { Variation }\end{array}$ & $\begin{array}{l}\text { miRNAs targ } \\
\text { submitted va } \\
\text { spike glycops } \\
\text { COV-2 seque }\end{array}$ \\
\hline $\begin{array}{l}\text { NCBI Reference Sequence: } \\
\text { NC_045512.2 }\end{array}$ & AATAACGCTACTAATGTTGTTATTAAATCTGTGAATTTCAATTTTGTAATGATCCATTTTTGGGTGTTTATTACCAAAAAACAACAAAAGTTGGATGGAAAGT & original & $\begin{array}{l}\text { miRNA Nam } \\
\text { 196a-1-3p } \\
\text { miRNA Sequ } \\
\text { - caacaac auu } \\
3^{\prime} \\
\text { (length }=21 \text { ) }\end{array}$ \\
\hline $\begin{array}{l}\text { Sample 23: } \\
\text { hCoV-19/Jordan/SR- } \\
\text { 042/2020|EPI_ISL_430000|2020- } \\
03-30\end{array}$ & $\begin{array}{l}\text { AATAACGCTACTAATGTTGTTATTAAATCTGTGAATTTCAATTTTGTAATGATCCATTTTTGGGTGTTTACCAAAAAACAACAAAAGTTGGATGGAAAGT } \\
\end{array}$ & $\begin{array}{l}\text { del } 432 \\
\text { TTA } \\
\text { del. Y144 }\end{array}$ & $\begin{array}{l}\text { miRNA Nam } \\
\text { 196a-1-3p } \\
\text { miRNA Sequu } \\
\text { - caacaacauu } \\
3^{\prime} \\
\text { (length }=21 \text { ) }\end{array}$ \\
\hline $\begin{array}{l}\text { NCBI Reference Sequence: } \\
\text { NC_045512.2 }\end{array}$ & GGAACAAATACTTCTAACCAGGTTGCTGTTCTTTATCAGGATGTTAACTGCACAGAAGTCCCTGTTGCTATTCATGCAGATCAACTTACTCCTACTTGGCGTGT & $\begin{array}{l}\text { Original } \\
1841 \mathrm{~A}\end{array}$ & $\begin{array}{l}\text { miRNA Nam } \\
\text { 4793-5p } \\
\text { miRNA Sequ } \\
- \\
\text { acauccugcuc } \\
-3 \text { ' (length = }\end{array}$ \\
\hline 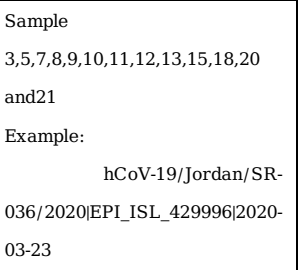 & GGAACAAATACTTCTAACCAGGTTGCTGTTCTTTATCAGGGTGTTAACTGCACAGAAGTCCCTGTTGCTATTCATGCAGATCAACTTACTCCTACTTGGCGTGT & $\begin{array}{l}1841 \mathrm{~A}>\mathrm{G} \\
\mathrm{D} 614 \mathrm{G}\end{array}$ & $\begin{array}{l}\text { miRNA Nam } \\
3620-3 p \\
\text { Previous Nar } \\
3620 \\
\text { miRNA Sequ } \\
\text { - ucacccugca } \\
\text { 3' (length = 2 }\end{array}$ \\
\hline $\begin{array}{l}\text { NCBI Reference Sequence: } \\
\text { NC_045512.2 }\end{array}$ & ACTGTGATGTTGTAATAGGAATTGTCAACAACACAGTTTATGATCCTTTGCAACCTGAATTAGACTCATTCAAGGAGGAGTTAGATAAATATTTTAAGAATCATACA & $\begin{array}{l}\text { Original } \\
3415 G\end{array}$ & $\begin{array}{l}\text { miRNA Nam } \\
548 \mathrm{~g}-3 \mathrm{p} \\
\text { Previous Nar } \\
548 \mathrm{~g} \\
\text { miRNA Sequ } \\
\text { - aaaacuguaa } \\
\text { 3' (length }=2 \\
\end{array}$ \\
\hline $\begin{array}{l}\text { Sample 21: } \\
\text { hCoV-19/Jordan/SR- } \\
\text { 033/2020|EPI_ISL_429993|2020- } \\
\text { 03-16 }\end{array}$ & ACTGTGATGTTGTAATAGGAATTGTCAACAACACAGTTTATTATCCTTTGCAACCTGAATTAGACTCATTCAAGGAGGAGTTAGATAAATATTTTAAGAATCATACA & $\begin{array}{l}3415 \mathrm{G}>\mathrm{T} \\
\mathrm{D} 1139 \mathrm{Y}\end{array}$ & $\begin{array}{l}\text { miRNA Nam } \\
548 g \text {-3p } \\
\text { Previous Nar } \\
548 g \\
\text { miRNA Sequ } \\
\text { - aaaacuguaa } \\
\text { 3' (length = } 2 \\
\end{array}$ \\
\hline $\begin{array}{l}\text { NCBI Reference Sequence: } \\
\text { NC_045512.2 }\end{array}$ & TCACCAGATGTTGATTTAGGTGACATCTCTGGCATTAATGCTTCAGTTGTAAACATTCAAAAAGAAATTGACCGCCTCAATGAGGTTGCCAAGAATTTAAATG & $\begin{array}{l}\text { Original } \\
3499 \mathrm{G}\end{array}$ & $\begin{array}{l}\text { miRNA Nam } \\
155-5 p \\
\text { Previous Nar } \\
155 \\
\text { miRNA Sequi } \\
- \\
\text { uuaaugcuaau } \\
-3 \text { ' (length }= \\
\end{array}$ \\
\hline $\begin{array}{l}\text { Sample 16: } \\
\text { hCoV-19/Jordan/SR- } \\
\text { 039/2020|EPI_ISL_429998|2020- } \\
\text { 03-28 }\end{array}$ & TCACCAGATGTTGATTTAAGTGACATCTCTGGCATTAATGCTTCAGTTGTAAACATTCAAAAAGAAATTGACCGCCTCAATGAGGTTGCCAAGAATTTAAATG & $\begin{array}{l}3499 \\
\text { G }>A \\
\text { G1167S }\end{array}$ & $\begin{array}{l}\text { miRNA Nam } \\
155-5 p \\
\text { Previous Nar } \\
155 \\
\text { miRNA Sequ } \\
- \\
\text { uuaaugcuaau } \\
-3 \text { ' (length }=\end{array}$ \\
\hline
\end{tabular}

\section{References}

1. Wu F, Zhao S, Yu B, Chen YM, Wang W, Song ZG, et al. A new coronavirus associated with human respiratory disease in China. Nature. 2020 Mar 12;579(7798):265-9.

2. Perlman S, Netland J. Coronaviruses post-SARS: Update on replication and pathogenesis. Vol. 7, Nature Reviews Microbiology. Nature Publishing Group; 2009. p. 439-50.

3. Trobaugh DW, Gardner CL, Sun C, Haddow AD, Wang E, Chapnik E, et al. RNA viruses can hijack vertebrate microRNAs to suppress innate immunity. Nature. 2014 Dec 18;506(7487):245-8.

4. Trobaugh DW, Klimstra WB. MicroRNA Regulation of RNA Virus Replication and Pathogenesis. Trends Mol Med. 2017 Jan 1;23(1):80-93. 
5. Peng S, Wang J, Wei S, Li C, Zhou K, Hu J, et al. Endogenous Cellular MicroRNAs Mediate Antiviral Defense against Influenza A Virus. Mol Ther - Nucleic Acids. 2018 Mar 2;10:361-75.

6. Morales L, Oliveros JC, Fernandez-Delgado R, tenOever BR, Enjuanes L, Sola I. SARS-CoV-Encoded Small RNAs Contribute to Infection-Associated Lung Pathology. Cell Host Microbe. 2017 Mar 8;21(3):344-55.

7. Chen Y, Wang X. miRDB: an online database for prediction of functional microRNA targets. Nucleic Acids Res. 2019;48:127-31.

8. Liu W, Wang X. Prediction of functional microRNA targets by integrative modeling of microRNA binding and target expression data. Genome Biol. 2019 Jan 22;20(1):18.

9. Oostra M, de Haan CAM, Rottier PJM. The 29-Nucleotide Deletion Present in Human but Not in Animal Severe Acute Respiratory Syndrome Coronaviruses Disrupts the Functional Expression of Open Reading Frame 8. J Virol. 2007 Dec 15;81(24):13876-88.

\section{Declarations}

The authors declare no competing interests. 